\title{
Correction to: Establishment and characterization of novel cell lines derived from six lepidopteran insects collected in the field
}

\author{
Kazuyo Watanabe ${ }^{1} \cdot$ Isao Kobayashi ${ }^{2} \cdot$ Masatsugu Hatakeyama $^{3} \cdot$ Takumi Kayukawa $^{1} \cdot$ Gaku Akiduki $^{1,4}$
}

Published online: 12 June 2020

(C) The Society for In Vitro Biology 2020

\section{Correction to: In Vitro Cellular \& Developmental Biology - Animal https://doi.org/10.1007/s11626-020-00438-5}

This article was originally published with the final word of the title, "field", omitted.

Also, the final sentence of the article was rendered incorrectly as "Each cell line established here has already been adapted to a commercial medium, Grace's insect medium, Supplemented"Grace's insect medium, Supplemented" is the commercial name of this medium. (Thermo Fisher Scientific), and all six cell lines will be distributed from the Genebank Project, NARO (https://www.gene.affrc.go.jp/distribution.php)."

The original article has been corrected.

The online version of the original article can be found at https://doi.org/ $10.1007 / \mathrm{s} 11626-020-00438-5$

Gaku Akiduki

akiduki@affrc.go.jp

1 Division of Insect Sciences, Institute of Agrobiological Sciences,

National Agriculture and Food Research Organization (NARO), 1-2,

Owashi, Tsukuba 305-8634, Japan

2 Division of Biotechnology, Institute of Agrobiological Sciences,

National Agriculture and Food Research Organization (NARO), 1-2,

Owashi, Tsukuba 305-8634, Japan

3 Division of Applied Genetics, Institute of Agrobiological Sciences,

National Agriculture and Food Research Organization (NARO), 1-2,

Owashi, Tsukuba 305-8634, Japan

4 Division of Agro-Environment Research, Kyushu Okinawa

Agricultural Research Center, National Agriculture and Food

Research Organization (NARO), 2421, Suya,

Koshi, Kumamoto 861-1192, Japan 